Research Article

\title{
$\beta$-Stability in $q$-th Moment of Neutral Impulsive Stochastic Functional Differential Equations with Markovian Switching
}

\author{
Lassaad Mchiri $\mathbb{D}^{1,2}$ and Mohamed Rhaima ${ }^{1,3}$ \\ ${ }^{1}$ Department of Statistics and Operations Research, College of Sciences, King Saud University, Riyadh 11451, Saudi Arabia \\ ${ }^{2}$ Department of Mathematics, Faculty of Sciences of Sfax, University of Sfax, Sfax, Tunisia \\ ${ }^{3}$ University of Tunis El Manar, Faculty of Sciences of Tunis, Department of Mathematics, Tunis, Tunisia
}

Correspondence should be addressed to Lassaad Mchiri; lmchiri.c@ksu.edu.sa

Received 30 March 2021; Accepted 24 April 2021; Published 8 May 2021

Academic Editor: Abdellatif Ben Makhlouf

Copyright ( 2021 Lassaad Mchiri and Mohamed Rhaima. This is an open access article distributed under the Creative Commons Attribution License, which permits unrestricted use, distribution, and reproduction in any medium, provided the original work is properly cited.

In this paper, we investigate the $\beta$-stability in $q$-th moment for neutral impulsive stochastic functional differential equations with Markovian switching (NISFDEwMS). Moreover, $\beta$-stability in $q$-th moment is studied by using the Lyapunov techniques and a new Razumikhin-type theorem to prove our result. Finally, we check the main result by a numerical example.

\section{Introduction}

The aim of stochastic differential equations is to provide a mathematical model for a differential equation disturbed by a random noise. Consider an ordinary differential equation of the form

$$
\mathrm{d} \sigma_{\tau}=a\left(\sigma_{\tau}\right) \mathrm{d} \tau
$$

An ordinary differential equation is used to describe the evolution of a physical system. If we take into account the random disturbances, we add a noise term, which will be of the form $g d W_{\tau}$, where $W$ denotes a Brownian motion and $g$, for this instant, is a constant which corresponds to the intensity of the noise. Then, we have a stochastic differential equation (SDE) of the form

$$
\mathrm{d} \sigma_{\tau}=a\left(\sigma_{\tau}\right) \mathrm{d} \tau+g d W_{\tau} .
$$

Moreover, we have the following integral form:

$$
\sigma_{\tau}=\sigma_{0}+\int_{0}^{\tau} a\left(\sigma_{t}\right) \mathrm{d} t+g W_{\tau}
$$

We generalize equation (3) by taking $g$ depending on the state of the system to an instant $\tau$ :

$$
\sigma_{\tau}=\sigma_{0}+\int_{0}^{\tau} a\left(\sigma_{t}\right) \mathrm{d} t+\int_{0}^{\tau} g\left(\sigma_{t}\right) \mathrm{d} W_{t}
$$

In view of the integral in $\mathrm{d} W_{t}$, this equation will depend on the theory of stochastic integral (see [1]).

One of the most important qualitative studies of SDE is the stability theory.

There are a lot of papers about the stability theory of different types of SDE (see [1-3]).

A special case of SDE is the neutral stochastic functional differential equations (NSFDE).

In the literature, there are many research papers on the stability theory of NSFDE (see [4-6]). Recently, stability of SFDE with Markovian switching (SFDEwMS) has received a lot of attention (see [7-11]).

One of the most important classes of SDE is the impulsive SDE (ISDE). In the last decades, the stability theory of ISDE takes much more attention (see [7, 11-13]).

To the best of our knowledge, there are a few papers on the $\beta$-stability or stability of SDE with general decay rate. In [14-16], the authors established the stability with general decay rate of stochastic functional differential equations with finite and infinite delay.

In the literature, there is no existing paper on the $\beta$-stability of NISFDEwMS. 
In this paper, we study the $\beta$-stability in $q$-th moment of NISFDEwMS. In view of the other related topics on NISFDEwMS, the main contributions of this paper are to obtain a new technique to study the $\beta$-stability in a hybrid impulsive neutral stochastic system, to develop a new Razumikhin-type theorem on the Lyapunov function to prove the $\beta$-stability of the system, and to propose new theories on asymptotic properties in $q$-th moment of NISFDEwMS. In this sense, our results generalize the paper [16] in the case of NISFDEwMS.

The paper is organized as follows. In Section 2, we cite some definitions and basic notions. In Section 3, we investigate the $\beta$-stability in $q$-th moment of NISFDEwMS. In Section 4 , one numerical example is given to show the interest of our main results.

\section{Preliminaries and Definitions}

The complete probability space is denoted by $\left\{\Omega, \mathbb{F},\left(\mathbb{F}_{\tau}\right)_{\tau \geq 0}, \mathbb{P}\right\}$, where $\left\{\mathbb{F}_{\tau}\right\}_{\tau \geq 0}$ is a filtration satisfying the usual conditions. $W(\tau)$ is an $m$-dimensional Brownian motion defined on the probability space. Let $l>0$ and $\mathscr{C}\left([-l, 0] ; \mathbb{R}^{i}\right)$ be the family of continuous functions $\psi$ from $[-l, 0]$ to $\mathbb{R}^{i}$ with the norm $\|\psi\|=\sup _{-l \leq b \leq 0}|\psi(b)|$, where $|z|=\sqrt{z^{T} z}$ for any $z \in \mathbb{R}^{i}$. If $B$ is a matrix, its trace norm is denoted by $|B|=\sqrt{\operatorname{trace}\left(B^{T} B\right)}$, where its norm is given by $\|B\|=\sup _{|z|=1}|B z|$.

Let $P \mathscr{C}\left([-l, 0] ; \mathbb{R}^{i}\right)=\left\{\phi:[-l, 0] \longrightarrow \mathbb{R}^{i} \mid \phi\left(t^{+}\right)=\phi(t)\right\}$, where $\phi\left(t^{-}\right)$exists and $\phi\left(t^{-}\right)=\phi(t)$ for all but at most a finite number of points $t$, with the uniform norm $\|\phi\|=\sup _{-l \leq b \leq 0}|\phi(b)|$, where $\phi\left(t^{+}\right)$and $\phi\left(t^{-}\right)$denote the right-hand and left-hand limits of $\phi(t)$ at $t$, respectively. For $q \geq 1$, let $\mathbb{L}^{q}\left((-\infty, 0], \mathbb{R}^{i}\right)$ be the family of all function $\mu:(-\infty, 0] \longrightarrow \mathbb{R}^{i}$ such that $\int_{-\infty}^{0}|\mu(\tau)|^{q} \mathrm{~d} \tau<\infty$.

Let $\left\{m(\tau), \tau \geq \tau_{0}\right\}$ be a right-continuous Markov chain on the probability space taking values in a finite state space $S=\{1,2,3, \ldots, N\}$ with $\Gamma=\left(\gamma_{j k}\right)_{\mathbb{N} \times \mathbb{N}}$ as a generator given by

$$
P(m(s+\Phi)=k \mid m(s)=j)= \begin{cases}\gamma_{j k} @+o(@), & \text { if } j \neq k, \\ 1+\gamma_{j j} @+o(@), & \text { if } j=k,\end{cases}
$$

while $\Phi>0$. Here, $\gamma_{j k} \geq 0$ is the transition rate from $j$ to $k$, if $j \neq k$, while

$$
\gamma_{j j}=-\sum_{j \neq k} \gamma_{j k}
$$

We consider the following NISFDEwMS:

$$
\begin{cases}d\left(\sigma(\tau)-G\left(\sigma_{\tau}\right)\right)=f_{1}\left(\sigma_{\tau}, \tau, m(\tau)\right) \mathrm{d} \tau+f_{2}\left(\sigma_{\tau}, \tau, m(\tau)\right) \mathrm{d} w(\tau), & \tau \geq 0, \\ \Delta \sigma\left(\tau_{p}\right)=I_{p}\left(\sigma\left(\tau_{p}\right), \sigma_{\tau_{p}}, \tau_{p}, m\left(\tau_{p}\right)\right), & p=1,2, \ldots, \\ \sigma(\tau)=\chi \in P \mathscr{C}\left([-l, 0] ; \mathbb{R}^{i}\right), & \tau \in[-l, 0],\end{cases}
$$

where $\sigma_{\tau}$ is defined by $\sigma_{\tau}(\theta)=\{\sigma(\tau+\theta),-l \leq \theta \leq 0\}$, which is a $P \mathscr{C}\left([-l, 0] ; \mathbb{R}^{i}\right)$-valued stochastic process, $\tau_{p} \geq 0$ are impulsive moments satisfying $\tau_{p}<\tau_{p+1}$ and $\lim _{\tau_{p}} \longrightarrow+\infty$ $\tau_{p}=+\infty$, and $\Delta \sigma\left(\tau_{p}\right)=\sigma\left(\tau_{p}^{+}\right)-\sigma\left(\tau_{p}\right)$ represents the jump in the state $\sigma$ at $\tau_{p}$ with $I_{p}$ determining the size of the jump. Furthermore, we assume that

$$
\begin{aligned}
& f_{1}: P \mathscr{C}\left([-l, 0] ; \mathbb{R}^{i}\right) \times[0,+\infty) \times S \longrightarrow \mathbb{R}^{i}, f_{2}: P \mathscr{C}\left([-l, 0] ; \mathbb{R}^{i}\right) \times[0,+\infty) \times S \longrightarrow \mathbb{R}^{n \times m}, \\
& G: P \mathscr{C}\left([-l, 0] ; \mathbb{R}^{i}\right) \longrightarrow \mathbb{R}^{i}, \\
& I_{p}: \mathbb{R}^{i} \times P \mathscr{C}\left([-l, 0] ; \mathbb{R}^{i}\right) \times[0,+\infty) \times S \longrightarrow \mathbb{R}^{i} .
\end{aligned}
$$

Let $C^{1,2}\left(\mathbb{R}^{i} \times[-l, 0] \times S, \mathbb{R}_{+}\right)$be the family of all nonnegative functions $V(\sigma, \tau, j)$ on $\mathbb{R}^{i} \times[-l, 0] \times S$, which are twice continuously differentiable with respect to $\sigma$ and once continuously differentiable with respect to $\tau$.
Define the operator $\mathscr{L} V: \mathbb{R}^{i} \times P \mathscr{C}\left([-l, 0] ; \mathbb{R}^{i}\right) \times \mathbb{R}_{+} \times$ $S \longrightarrow \mathbb{R}$ by (see [9])

$$
\begin{aligned}
\mathscr{L} V(\sigma, \psi, \tau, j)= & V_{\tau}(\sigma-G(\psi), \tau, j)+V_{\sigma}(\sigma-G(\psi), \tau, j) f_{1}(\psi, \tau, j) \\
& +\frac{1}{2} \operatorname{trace}\left(f_{2}^{T}(\psi, \tau, j) V_{\sigma \sigma}(\sigma-G(\psi), \tau, j) f_{2}(\psi, \tau, j)\right)+\sum_{k=1}^{N} \gamma_{j k} V(\sigma-G(\psi), \tau, k),
\end{aligned}
$$


where

$$
\begin{aligned}
V_{\tau}(\sigma, \tau, j) & =\frac{\partial V(\sigma, \tau, j)}{\partial \tau}, V_{\sigma}(\sigma, \tau, j)=\left(\frac{\partial V(\sigma, \tau, j)}{\partial \sigma_{1}}, \ldots, \frac{\partial V(\sigma, \tau, j)}{\partial \sigma_{i}}\right), \\
V_{\sigma \sigma}(\sigma, \tau, j) & =\left(\frac{\partial^{2} V(\sigma, \tau, j)}{\partial \sigma_{m} \partial \sigma_{n}}\right)_{i \times i} .
\end{aligned}
$$

Throughout this paper, we assume that $f_{1}, f_{2}, G$, and $I_{p}$ are smooth enough to ensure the global existence and uniqueness of solutions for all $\tau \geq 0$. For any $\chi \in L_{\mathbb{F}_{0}}^{q}\left([-l, 0] ; \mathbb{R}^{i}\right)$, there is a unique solution satisfying (7), which is continuous on the left-hand side and have a finite limit on the right-hand side. We assume that $f(0, \tau, j)=0$, $g(0, \tau, i)=0$, and $I_{p}(0,0, \tau, j)=0$, where $\forall \tau \geq 0, j \in S$, and $p=1,2, \ldots$, which implies that 0 is an equilibrium solution.

Definition 1. The function $\beta: \mathbb{R}_{+} \longrightarrow(0, \infty)$ is said to be $\beta$-type function if it satisfies the following conditions:

(i) It is continuous and nondecreasing in $\mathbb{R}_{+}$and continuously differentiable in $\mathbb{R}_{+}$

(ii) $\beta(0)=1, \quad \lim _{\tau \longrightarrow \infty} \beta(\tau)=\infty$, and $M_{\beta}=\sup _{t>0}$ $\left|\beta^{\prime}(\tau) / \beta(\tau)\right|<\infty$

(iii) For any $s, \tau \geq 0, \beta(\tau) \leq \beta(s) \beta(\tau-s)$

Definition 2. System (7) is said to be $\beta$-stable in $q$-th moment $(\beta$-s.q.m), if there exists a function $\beta$ satisfying Definition 1 and positive constants $b_{1}$ and $\eta$ such that, for any $\chi \in P \mathscr{C}\left([-l, 0] ; \mathbb{R}^{i}\right) \cap \mathbb{L}^{q}\left((-\infty, 0], \mathbb{R}^{i}\right)$,

$$
\mathbb{E}\left(|\sigma(\tau)|^{q}\right) \leq b_{1} \mathbb{E}\left(\|\chi\|^{q}\right) \beta^{-\eta}(\tau), \quad \tau \geq 0,
$$

which is equivalent to

$$
\limsup _{\tau \longrightarrow \infty} \frac{\ln \mathbb{E}\left(|\sigma(\tau)|^{q}\right)}{\ln \beta(\tau)} \leq-\eta,
$$

when $q=2$, it is said to be $\beta$-stable in mean square.

\section{Main Results}

Theorem 1. Assume that $V \in C^{1,2}\left(\mathbb{R}^{i} \times[-l, \infty) \times S, \mathbb{R}_{+}\right)$, constants $q>1, \varepsilon_{1}>0, \varepsilon_{2}>0, a_{j, p} \geq 0, \bar{a}_{j, p} \geq 0, a_{j, p}^{2}+\bar{a}_{j, p}^{2} \neq 0$, $\gamma_{1}>0, \gamma_{2}>0, \alpha_{j} \geq 0, j \in S$, and $p=1,2, \ldots$, and a function $\beta$ satisfying Definition 1 such that

(i) $\varepsilon_{1}|\sigma|^{q} \leq V(\sigma, \tau, j) \leq \varepsilon_{2}|\sigma|^{q} \quad$ and $\quad \forall(\sigma, \tau, j) \in \mathbb{R}^{i} \times$ $[-l,+\infty) \times S$.

(ii) $\forall \tau \in\left(\tau_{p-1}, \tau_{p}\right]$ and $j \in S$ satisfying

$\mathbb{E}(\mathscr{L} V(\psi(0), \psi, \tau, j)) \leq \alpha_{j} \frac{\beta^{\prime}(\tau)}{\beta(\tau)} \mathbb{E}(V(\psi(0)-G(\psi), \tau, j)$,

where $\forall \tau \geq 0$ and those $\quad \psi \in P \mathscr{C}([-l, 0]$; $\left.\mathbb{R}^{i}\right) \cap \mathbb{L}^{q}\left((-\infty, 0], \mathbb{R}^{i}\right)$ satisfying

$$
\mathbb{E}\left(\min _{1 \leq j \leq N} V(\psi(\theta), \tau+\theta, j)\right) \leq s \beta^{\gamma_{2}}(l) \mathbb{E}\left(\max _{1 \leq j \leq N} V(\psi(0)-G(\psi), \tau, j)\right) .
$$

(iii) $\forall j \in S$ satisfying

$$
\begin{aligned}
\mathbb{E} & \left(V\left(\psi(0)-G(\psi)+I_{p}\left(\psi(0), \psi(\theta), \tau_{p}, j\right), \tau_{k}^{+}, j\right)\right) \\
& \leq a_{j, p} \mathbb{E}\left(V\left(\psi(0)-G(\psi), \tau_{p}, j\right)\right) \\
& +\bar{a}_{j, p} \sup _{l \leq \theta \leq 0} \mathbb{E}\left(V\left(\psi(\theta)-G(\psi), \tau_{p}, j,\right)\right) .
\end{aligned}
$$

(iv) $\sup _{1 \leq p<\infty}\left\{\tau_{p}-\tau_{p-1}\right\} \leq \gamma_{1}$.

(v) For any $j \in S, \gamma_{2}+\alpha_{j} \leq \ln (s) / \gamma_{1}$.
Then, the solution of system (7) is $\beta$-stable in $p$-th moment, where $2^{q-1} k^{q} \beta^{\gamma_{2}}(l)<1$ and $s=1 / \max _{j \in S, 1 \leq p<\infty}$ $\left(a_{j, p}+\bar{a}_{j, p} \beta^{\gamma_{2}}(l)\right)>1$.

Proof. For any $\chi \in P \mathscr{C}\left([-l, 0] ; \mathbb{R}^{i}\right) \cap \mathbb{L}^{q}\left((-\infty, 0], \mathbb{R}^{i}\right)$, let $\sigma(\tau)=\sigma(\tau, \chi)$ be the solution of (7) and $m(\tau)=m(0)=m_{0}$, where $\forall \tau \in[-l, 0]$. Let $\delta>0$ be sufficiently small such that $\tau+\delta \in\left(\tau_{p-1}, \tau_{p}\right)$. By using Itô formula, we obtain 


$$
\mathbb{E}\left(V\left(\sigma(\tau+\delta)-G\left(\sigma_{\tau+\delta}\right), \tau+\delta, m(\tau+\delta)\right)\right)=\mathbb{E}\left(V\left(\sigma(\tau)-G\left(\sigma_{\tau}\right), \tau, m(\tau)\right)\right)+\int_{\tau}^{\tau+\delta} \mathbb{E}\left(\mathscr{L} V\left(\sigma_{t}, t, m(t)\right) \mathrm{d} t\right)
$$

Let $\delta \longrightarrow 0$, we can derive that, for $\tau \in\left(\tau_{p-1}, \tau_{p}\right]$, $D^{+} E\left(V\left(\sigma(\tau)-G\left(\sigma_{\tau}\right), \tau, m(\tau)\right)\right)=\mathbb{E}\left(\mathscr{L} V\left(\sigma(\tau), \sigma_{\tau}, \tau, m(\tau)\right)\right)$,

where

$$
D^{+} g(\tau)=\limsup _{h \longrightarrow 0^{+}} \frac{g(\tau+h)-g(\tau)}{h} .
$$

Let $S(\tau)=\beta^{\gamma_{2}}(\tau) \mathbb{E}\left(V\left(\sigma(\tau)-G\left(\sigma_{\tau}\right), \tau, j\right)\right)$, then we have for $\tau \in\left(\tau_{p-1}, \tau_{p}\right]$,

$$
\begin{aligned}
D^{+} S(\tau)= & \gamma_{2} \frac{\beta^{\prime}(\tau)}{\beta(\tau)} \beta^{\gamma_{2}}(\tau) \mathbb{E}\left(V\left(\sigma(\tau)-G\left(\sigma_{\tau}\right), \tau, j\right)\right) \\
& +\beta^{\gamma_{2}}(\tau) \mathbb{E}\left(\mathscr{L} V\left(\sigma(\tau), \sigma_{\tau}, \tau, j\right)\right) .
\end{aligned}
$$

By (iii), we obtain

$$
\begin{aligned}
S\left(\tau_{p}^{+}\right) & =\beta^{\gamma_{2}}\left(\tau_{p}\right)\left(V\left(\sigma\left(\tau_{p}^{+}\right)-G\left(\sigma_{\tau_{p}^{+}}\right), \tau_{p}^{+}, j\right)\right) \\
& \leq a_{j, p} S\left(\tau_{p}\right)+\bar{a}_{j, p} \beta^{\gamma_{2}}(l) \sup _{-l \leq \theta \leq 0} S\left(\tau_{p}+\theta\right) .
\end{aligned}
$$

Let $C>0$ such that

$$
\sup _{-l \leq \theta \leq 0} S(\theta)<\frac{C}{s}
$$

We can claim that, for $\tau \geq-l$,

$$
S(\tau)<C \text {. }
$$

By (19), it is clear to see that $S(\tau)<C$ for $\tau \in[-l, 0]$. We will prove that

$$
S(\tau)<C, \quad \tau \in\left(0, \tau_{1}\right]
$$

Otherwise, there exists $\bar{\tau} \in\left(0, \tau_{1}\right]$ satisfying $S(\bar{\tau})=C$ and $S(\tau)<C$, for $\tau \in(-l, \bar{\tau})$. By the continuity of $S(\tau)$ in $\left[0, \tau_{1}\right]$, there exists $\tilde{\tau} \in(0, \bar{\tau})$ satisfying

$$
S(\tilde{\tau})=\frac{C}{s}, S(\tau)>\frac{C}{s}, \quad \tau \in(\tilde{\tau}, \bar{\tau}] .
$$

For $\tau \in[\tilde{\tau}, \bar{\tau}]$ and $\theta \in[-l, 0]$, we obtain

$$
S(\tau)>S(\tau+\theta)
$$

Thus, we have

$$
\begin{aligned}
s \beta^{\gamma_{2}}(l) \mathbb{E}\left(\max _{1 \leq j \leq N} V(\psi(0)-G(\psi), \tau, j)\right) & \geq s \beta^{-\gamma_{2}}(\tau) \beta^{\gamma_{2}}(l) S(\tau)>\beta^{-\gamma_{2}}(\tau) \beta^{\gamma_{2}}(l) S(\tau+\theta) \\
& \geq \mathbb{E}\left(\min _{1 \leq j \leq N} V\left(\sigma(\tau+\theta)-G\left(\sigma_{\tau+\theta}\right), \tau+\theta, j\right)\right) .
\end{aligned}
$$

Combining (26) with (19) and (ii), for $\tau \in[\tilde{\tau}, \bar{\tau}]$, we have

$$
\begin{aligned}
D^{+} S(\tau) & \leq \gamma_{2} M_{\beta} \beta^{\gamma_{2}}(\tau) \mathbb{E}\left(V\left(\sigma(\tau)-G\left(\sigma_{\tau}\right), \tau, j\right)\right)+\alpha_{j} M_{\beta} \beta^{\gamma_{2}}(\tau) \mathbb{E}\left(V\left(\sigma(\tau)-G\left(\sigma_{\tau}\right), \tau, j\right)\right) \\
& =M_{\beta} \beta^{\gamma_{2}}(\tau)\left(\gamma_{2}+\alpha_{j}\right) \mathbb{E}\left(V\left(\sigma(\tau)-G\left(\sigma_{\tau}\right), \tau, j\right)\right) \\
& =M_{\beta}\left(\gamma_{2}+\alpha_{j}\right) S(\tau) .
\end{aligned}
$$

Then,

$$
\begin{aligned}
C & =S(\bar{\tau}) \leq S(\widetilde{\tau}) e^{M_{\beta}\left(\gamma_{2}+\alpha_{j}\right)(\bar{\tau}-\tilde{\tau})} \\
& =\frac{C}{s} e^{M_{\beta}\left(\gamma_{2}+\alpha_{j}\right)(\bar{\tau}-\tilde{\tau})} \\
& <\frac{C}{s} e^{M_{\beta}\left(\gamma_{2}+\alpha_{j}\right) \tau_{1}} \\
& \leq C .
\end{aligned}
$$

This is a contradiction. Therefore, (23) holds. By (iii), we can derive

$$
S\left(\tau_{1}^{+}\right) \leq a_{j, 1} S\left(\tau_{1}\right)+\bar{a}_{j, 1} \beta^{\gamma_{2}}(l) \sup _{-l \leq \theta \leq 0} S\left(\theta+\tau_{1}\right)<\frac{C}{s}<C
$$

Now, we will prove that

$$
S(\tau)<C, \quad \tau \in\left(\tau_{1}, \tau_{2}\right] .
$$

Suppose that (30) does not hold, there exists $\bar{\tau}_{1} \in\left(\tau_{1}, \tau_{2}\right]$ such that

$$
S\left(\bar{\tau}_{1}\right)=C, S\left(\bar{\tau}_{1}\right)<C, \quad \tau \in\left[-l, \bar{\tau}_{1}\right] .
$$

By the continuity of $S(\tau)$ in $\left(\tau_{1}, \tau_{2}\right]$, there exists $\tilde{\tau}_{1} \in\left(\tau_{1}, \bar{\tau}_{1}\right)$ satisfying 


$$
S\left(\widetilde{\tau}_{1}\right)=\frac{C}{s}, S(\tau)>\frac{C}{s}, \quad \tau \in\left(\widetilde{\tau}_{1}, \bar{\tau}_{1}\right]
$$

For $\tau \in\left[\widetilde{\tau}_{1}, \bar{\tau}_{1}\right]$ and $\theta \in[-l, 0]$, we have

$$
s S(\tau)>S(\tau+\theta) .
$$

Using (19) and (ii), for $\tau \in\left[\widetilde{\tau}_{1}, \bar{\tau}_{1}\right]$, we obtain

$$
D^{+} S(\tau) \leq M_{\beta}\left(\gamma_{2}+\alpha_{j}\right) S(\tau)
$$

Thus, we obtain

$$
\begin{aligned}
C & =S\left(\bar{\tau}_{1}\right) \leq S\left(\widetilde{\tau}_{1}\right) e^{M_{\beta}\left(\gamma_{2}+\alpha_{j}\right)\left(\bar{\tau}_{1}-\tilde{\tau}_{1}\right)} \\
& =\frac{C}{s} e^{M_{\beta}\left(\gamma_{2}+\alpha_{j}\right)\left(\bar{\tau}_{1}-\tilde{\tau}_{1}\right)} \\
& <\frac{C}{s} e^{M_{\beta}\left(\gamma_{2}+\alpha_{j}\right)\left(\tau_{2}-\tau_{1}\right)} \\
& \leq C .
\end{aligned}
$$

This is a contradiction. Then, (30) holds. By induction, we can show that, for $p=1,2, \ldots$,

$$
S(\tau)<C, \quad \tau \in\left(\tau_{p-1}, \tau_{p}\right] .
$$

Hence, we can derive for $\tau \geq-l$,

$$
S(\tau)<C .
$$

By (i) and (37), we obtain

$$
\begin{aligned}
\varepsilon_{1} \mathbb{E}\left|\sigma(\tau)-G\left(\sigma_{\tau}\right)\right|^{q} & \leq \mathbb{E}\left(V\left(\sigma(\tau)-G\left(\sigma_{\tau}\right), \tau, j\right)\right) \\
& \leq C \beta^{-\gamma_{2}}(\tau),
\end{aligned}
$$

which implies that

$$
\beta^{\gamma_{2}}(\tau) \mathbb{E}\left|\sigma(\sigma)-G\left(\sigma_{\tau}\right)\right|^{q} \leq \frac{C}{\epsilon_{1}} .
$$

On the other hand, for any $T>0$, we have

$$
\begin{aligned}
\sup _{0 \leq \tau \leq T}\left(\beta^{\gamma_{2}}(\tau) \mathbb{E}|\sigma(\tau)|^{q}\right) & \leq 2^{q-1} \sup _{0 \leq \tau \leq T}\left(\beta^{\gamma_{2}}(\tau) \mathbb{E}\left|\sigma(\tau)-G\left(\sigma_{\tau}\right)\right|^{q}\right)+2^{q-1} \sup _{0 \leq \tau \leq T}\left(\beta^{\gamma_{2}}(\tau) \mathbb{E}\left|G\left(\sigma_{\tau}\right)\right|^{q}\right) \\
& \leq 2^{q-1} \sup _{0 \leq \tau \leq T}\left(\beta^{\gamma_{2}}(\tau) \mathbb{E}\left|\sigma(\tau)-G\left(\sigma_{\tau}\right)\right|^{q}\right)+2^{q-1} k^{q} \sup _{0 \leq \tau \leq T} \beta^{\gamma_{2}}(\tau)\left(\mathbb{E}\left|\sigma_{\tau}\right|^{q}\right) \\
& \leq 2^{q-1} \sup _{0 \leq \tau \leq T}\left(\beta^{\gamma_{2}}(\tau) \mathbb{E}\left|\sigma(\tau)-G\left(\sigma_{\tau}\right)\right|^{q}\right)+2^{q-1} k^{q} \sup _{-l \leq \tau \leq T}\left(\beta^{\gamma_{2}}(\tau-\theta) \mathbb{E}|\sigma(\tau)|^{q}\right) \\
& \leq 2^{q-1} \frac{C}{\epsilon_{1}}+2^{q-1} k^{q} \sup _{-l \leq \tau \leq 0}\left(\beta^{\gamma_{2}}(\tau) \beta^{\gamma_{2}}(-\theta) \mathbb{E}|\sigma(\tau)|^{q}\right)+2^{q-1} k^{q} \sup _{0 \leq \tau \leq T}\left(\beta^{\gamma_{2}}(\tau) \beta^{\gamma_{2}}(-\theta) \mathbb{E}|\sigma(\tau)|^{q}\right) \\
& \leq 2^{q-1} \frac{C}{\epsilon_{1}}+2^{q-1} k^{q} \beta^{\gamma_{2}}(l) \beta^{\gamma_{2}}(0) \mathbb{E}\|\chi\|^{q}+2^{q-1} k^{q} \beta^{\gamma_{2}}(l) \sup _{0 \leq \tau \leq T}\left(\beta^{\gamma_{2}}(\tau) \mathbb{E}|\sigma(\tau)|^{q}\right)
\end{aligned}
$$

Then,

$$
\sup _{0 \leq \tau \leq T}\left(\beta^{\gamma_{2}}(\tau) \mathbb{E}|\sigma(\tau)|^{q}\right) \leq \frac{1}{1-2^{q-1} k^{q} \beta^{\gamma_{2}}(l)} \times 2^{q-1}\left(\frac{C}{\varepsilon_{1}}+k^{q} \beta^{\gamma_{2}}(l) \mathbb{E}\|\chi\|^{q}\right) .
$$

Letting $T \longrightarrow+\infty$, we have

$$
\sup _{0 \leq \tau \leq+\infty}\left(\beta^{\gamma_{2}}(\tau) \mathbb{E}|\sigma(\tau)|^{q}\right) \leq C_{1}
$$

where

$$
C_{1}=\frac{1}{1-2^{q-1} k^{q} \beta^{\gamma_{2}}(l)} \times 2^{q-1}\left(\frac{C}{\epsilon_{1}}+k^{q} \beta^{\gamma_{2}}(l) \mathbb{E}\|\chi\|^{q}\right) .
$$

This implies that, for all $\tau \geq 0$,

$$
\mathbb{E}|\sigma(\tau)|^{q} \leq C_{1} \beta^{-\gamma_{2}}(\tau) .
$$

Therefore,

$$
\limsup _{\tau \longrightarrow \infty} \frac{\ln \mathbb{E}\left(|\sigma(\tau)|^{q}\right)}{\ln \beta(\tau)} \leq-\gamma_{2}
$$

as desired.

Theorem 2. Assume that $V \in C^{1,2}\left(\mathbb{R}^{i} \times[-l, \infty) \times S, \mathbb{R}_{+}\right)$, constants $q>1, \varepsilon_{1}>0, \varepsilon_{2}>0, a_{j, p} \geq 0, \bar{a}_{j, p} \geq 0, a_{j, p}^{2}+\bar{a}_{j, p}^{2} \neq 0$, $\gamma>0, \lambda>0, \mu_{j}>0, j \in S$, and $p=1,2, \ldots$, and a function $\beta$ satisfying Definition 1 such that

(i) $\varepsilon_{1}|\sigma|^{q} \leq V(\sigma, \tau, j) \leq \varepsilon_{2}|\sigma|^{q} \quad$ and $\quad \forall(\sigma, \tau, j) \in \mathbb{R}^{i} \times$ $[-l,+\infty) \times S$.

(ii) $\forall \tau \in\left(\tau_{p-1}, \tau_{p}\right]$ and $j \in S$ satisfying 
$\mathbb{E}(\mathscr{L} V(\psi(0), \psi, \tau, j)) \leq-\mu_{j} \frac{\beta^{\prime}(\tau)}{\beta(\tau)} \mathbb{E}(V(\psi(0)-G(\psi), \tau, j))$,

(46) where $\forall \tau \geq 0$ and those $\psi \in P \mathscr{C}\left([-l, 0] ; \mathbb{R}^{i}\right) \cap$ $\mathbb{L}^{q}\left((-\infty, 0], \mathbb{R}^{i}\right)$ satisfying

$$
\mathbb{E}\left(\min _{1 \leq j \leq N} V(\psi(\theta), \tau+\theta, j)\right) \leq s \beta^{\gamma}(l) \mathbb{E}\left(\max _{1 \leq j \leq N} V(\psi(0)-G(\psi), \tau, j)\right) .
$$

(iii) $\forall j \in S$ satisfying

$$
\begin{aligned}
& \mathbb{E}\left(V\left(\psi(0)-G(\psi)+I_{p}\left(\psi(0), \psi(\theta), \tau_{p}, j\right), \tau_{p}^{+}, j\right)\right) \\
& \leq a_{j, p} \mathbb{E}\left(V\left(\psi(0)-G(\psi), \tau_{p}, j\right)\right)+\bar{a}_{j, p} \sup _{-l \leq \theta \leq 0} \mathbb{E}\left(V\left(\psi(\theta)-G(\psi(\theta)), \tau_{p}, j,\right)\right) .
\end{aligned}
$$

(iv) $\inf _{1 \leq p<\infty}\left\{\tau_{p}-\tau_{p-1}\right\} \geq \lambda$.

(v) For any $j \in S, \mu_{j}-\gamma>\ln (s) / \lambda$.

Then, the solution of system (7) is $\beta$-stable in $q$-th moment, where $2^{q-1} k^{q} \beta^{\gamma}(l)<1$ and $s=1 / \max _{j \in S, 1 \leq p<\infty}$ $\left(a_{j, p}+\bar{a}_{j, p} \beta^{\gamma}(l)\right)>1$.

Proof. For any $\chi \in P \mathscr{C}\left([-l, 0] ; \mathbb{R}^{i}\right) \cap \mathbb{L}^{q}\left((-\infty, 0], \mathbb{R}^{i}\right)$, let $\sigma(\tau)=\sigma(\tau, \chi)$ be the solution of $(7)$ and $m(\tau)=m(0)=m_{0}$, where $\forall \tau \in[-l, 0]$. Since $(v)$ is satisfied, we can choose $\delta>0$ sufficiently small such that

$$
\mu_{j}-\gamma \geq \frac{\ln (s+\delta)}{\lambda}, \quad \forall j \in S .
$$

Let

$$
S(\tau)=\beta^{\gamma}(\tau) \mathbb{E}\left(V\left(\sigma(\tau)-G\left(\sigma_{\tau}\right), \tau, j\right)\right) .
$$

For $\tau \in\left(\tau_{p-1}, \tau_{p}\right]$, we obtain

$$
\begin{aligned}
D^{+} S(\tau)= & \gamma \frac{\beta^{\prime}(\tau)}{\beta(\tau)} \beta^{\gamma}(\tau) \mathbb{E}\left(V\left(\sigma(\tau)-G\left(\sigma_{\tau}\right), \tau, j\right)\right) \\
& +\beta^{\gamma}(\tau) \mathbb{E}\left(\mathscr{L} V\left(\sigma(\tau), \sigma_{\tau}, \tau, j\right)\right) .
\end{aligned}
$$

By (iii), we can derive

$$
\begin{aligned}
S\left(\tau_{p}^{+}\right) & =\beta^{\gamma}\left(\tau_{p}\right) \mathbb{E}\left(V\left(\sigma\left(\tau_{p}^{+}\right)-G\left(\sigma_{\tau_{p}^{+}}\right), \tau_{p}^{+}, j\right)\right) \\
& \leq a_{j, p} S\left(\tau_{p}\right)+\bar{a}_{j, p} \beta^{\gamma}(l) \sup _{-l \leq \theta \leq 0}\left(S\left(\tau_{p}+\theta\right)\right) .
\end{aligned}
$$

Let $C>0$ such that

$$
\sup _{-l \leq \theta \leq 0} S(\theta)<\frac{C}{s+\delta} .
$$

We will prove that, for $\tau \geq-l$,

$$
S(\tau)<C .
$$

Using (51), it is obvious to see that

$$
S(\tau)<C, \quad \text { for } \tau \in[-l, 0] .
$$

Now, we will prove that

$$
S(\tau)<C, \quad \text { for } \tau \in\left(0, \tau_{1}\right] .
$$

Otherwise, there exists $\bar{\tau} \in\left(0, \tau_{1}\right]$ satisfying $S(\bar{\tau})=C$ and $S(\tau)<C$, for $\tau \in(-l, \bar{\tau})$. By the continuity of $S(\tau)$ in $\left[0, \tau_{1}\right]$, there exists $\widetilde{\tau} \in[0, \bar{\tau})$ satisfying

$$
S(\widetilde{\tau})=\frac{C}{s+\delta}, S(\tau)>\frac{C}{s+\delta}, \quad \tau \in(\widetilde{\tau}, \bar{\tau}] .
$$

For $\tau \in\left[\widetilde{\tau}_{\delta}, \bar{\tau}\right]$ and $\theta \in[-l, 0]$, we obtain

$$
(s+\delta) S(\tau)>S(\tau+\theta) .
$$

Thus,

$$
\begin{aligned}
(s+\delta) \beta^{\gamma}(l) \mathbb{E}\left(\max _{1 \leq j \leq N}(V(\psi(0)-G(\psi), \tau, j))\right) & \geq(s+\delta) \beta^{-\gamma}(\tau) \beta^{\gamma}(l) S(\tau)>\beta^{-\gamma}(\tau) \beta^{\gamma}(l) S(\tau+\theta) \\
& \geq \mathbb{E}\left(\min _{1 \leq j \leq N}\left(V\left(\sigma(\tau+\theta)-G\left(\sigma_{\tau+\theta}\right), \tau+\theta, j\right)\right)\right) .
\end{aligned}
$$



obtain

Combining (59) with (51) and (ii), for $\tau \in[\tilde{\tau}, \bar{\tau}]$, we

$$
\begin{aligned}
D^{+} S(\tau) & \leq \gamma M_{\beta} \beta^{\gamma}(\tau) \mathbb{E}\left(V\left(\sigma(\tau)-G\left(\sigma_{\tau}\right), \tau, j\right)\right)-\mu_{j} M_{\beta} \beta^{\gamma}(\tau) \mathbb{E}\left(V\left(\sigma(\tau)-G\left(\sigma_{\tau}\right), \tau, j\right)\right) \\
& =M_{\beta}\left(\gamma-\mu_{j}\right) \beta^{\gamma}(\tau) \mathbb{E}\left(V\left(\sigma(\tau)-G\left(\sigma_{\tau}\right), \tau, j\right)\right) \\
& =M_{\beta}\left(\gamma-\mu_{j}\right) S(\tau) \leq 0 .
\end{aligned}
$$

Then,

$$
C=S(\bar{\tau}) \leq S(\widetilde{\tau})=\frac{C}{s+\delta}<C,
$$

which is a contradiction. Hence, (56) holds. Now, we will prove that

$$
S\left(\tau_{1}\right) \leq \frac{C}{s+\delta}
$$

If it is not satisfied, we have $S\left(\tau_{1}\right)>C / s+\delta$. By the continuity of $S(\tau)$ in $\left[0, \tau_{1}\right]$, there exists $\tau^{*} \in\left[0, \tau_{1}\right)$ satisfying

$$
S\left(\tau_{\delta}^{*}\right)=\frac{C}{s+\delta}, S(\tau)>\frac{C}{s+\delta}, \quad \tau \in\left(\tau^{*}, \tau_{1}\right] .
$$

For $\tau \in\left[\tau^{*}, \tau_{1}\right]$ and $\theta \in[-l, 0]$, we obtain

$$
(s+\delta) S(\tau)>S(\tau+\theta) \text {. }
$$

By (51) and (ii), for $\tau \in\left[\tau^{*}, \tau_{1}\right]$ and $\theta \in[-l, 0]$, we have

$$
D^{+} S(\tau) \leq M_{\beta}\left(\gamma-\mu_{j}\right) S(\tau) \leq 0 .
$$

Then,

$$
S\left(\tau_{1}\right) \leq S\left(\tau^{*}\right)=\frac{C}{s+\delta} .
$$

This is a contradiction. Using (52), (56), and (62), it follows that

$$
\begin{aligned}
S\left(\tau_{1}^{+}\right) & \leq a_{j, 1} S\left(\tau_{1}\right)+\bar{a}_{j, 1} \beta^{\gamma}(l) \sup _{-l \leq \theta \leq 0} S\left(\tau_{1}+\theta\right) \\
& \leq \frac{a_{j, 1}+\bar{a}_{j, 1} \beta^{\gamma}(l)}{s+\delta} C<C .
\end{aligned}
$$

Moreover, we can show that

$$
S(\tau)<C, \quad \forall \tau \in\left(\tau_{1}, \tau_{2}\right] .
$$

Indeed, there is $\bar{\tau}_{1} \in\left(\tau_{1}, \tau_{2}\right]$ satisfying

$$
S\left(\bar{\tau}_{1}\right)=C, S(\tau)<C, \quad \tau \in\left[-l, \bar{\tau}_{1}\right) .
$$

There exists $S(\tau)>C / s+\delta$ for $\tau \in\left(\tau_{1}, \bar{\tau}_{1}\right)$. Thus, $(s+$ $\delta) S(\tau)>S(\tau+\theta)$ for $\tau \in\left(\tau_{1}, \bar{\tau}_{1}\right)$ and $\theta \in[-l, 0]$. Then, by (51) and (ii), for $\tau \in\left(\tau_{1}, \bar{\tau}_{1}\right)$, we obtain

$$
D^{+} S(\tau) \leq M_{\beta}\left(\gamma-\mu_{j}\right) S(\tau) \leq 0,
$$

which implies that

$$
S\left(\bar{\tau}_{1}\right) \leq S\left(\tau_{1}^{+}\right)<C,
$$

which is a contradiction. If there is $\overline{\bar{\tau}}_{1} \in\left(\tau_{1}, \bar{\tau}_{1}\right)$ satisfying $S\left(\overline{\bar{\tau}}_{1}\right) \leq C / s+\delta$. By the continuity of $S(\tau)$ in $\left(\tau_{1}, \tau_{2}\right]$, there exists $\overline{\overline{\tau_{1}^{*}}} \in\left[\overline{\bar{\tau}}_{1}, \bar{\tau}_{1}\right)$ satisfying

$$
S\left(\overline{\overline{\tau_{1}^{*}}}\right)=\frac{C}{s+\delta}, S(\tau)>\frac{C}{s+\delta}, \quad \tau \in\left(\overline{\overline{\tau_{1}^{*}}}, \bar{\tau}_{1}\right) .
$$

Thus, for $\tau \in\left[\overline{\overline{\tau_{1}^{*}}}, \bar{\tau}_{1}\right]$ and $\theta \in[-l, 0]$,

$$
(s+\delta) S(\tau)>S(\tau+\theta) .
$$

Then, for

$$
D^{+} S(\tau) \leq M_{\beta}\left(\gamma-\mu_{j}\right) S(\tau) \leq 0,
$$

we have

$$
S\left(\bar{\tau}_{1}\right) \leq S\left(\overline{\overline{\tau_{1}^{*}}}\right)=\frac{C}{s+\delta}<C
$$

This is a contradiction. Hence, we can see that $S\left(\tau_{2}\right) \leq C / s+\delta$. If this is false, we have $S\left(\tau_{2}\right)>C / s+\delta$. To prove this claim, there are two cases to be presented:

Case 1: for $\tau \in\left(\tau_{1}, \tau_{2}\right], S(\tau)>C / s+\delta$. By (53), (56), and (69), we have for $\tau \in\left(\tau_{1}, \tau_{2}\right]$ and $\theta \in[-l, 0]$,

$$
(s+\delta) S(\tau)>S(\tau+\theta) \text {. }
$$

Thus, by (51) and (ii), for $\tau \in\left(\tau_{1}, \tau_{2}\right]$,

$$
D^{+} S(\tau) \leq M_{\beta}\left(\gamma-\mu_{j}\right) S(\tau) \text {. }
$$

Then,

$$
\begin{aligned}
S\left(\tau_{2}\right) & \leq S\left(\tau_{1}^{+}\right) e^{M_{\beta}\left(\gamma-\mu_{j}\right)\left(\tau_{2}-\tau_{1}\right)} \\
& <C e^{M_{\beta}\left(\gamma-\mu_{j}\right)\left(\tau_{2}-\tau_{1}\right)} \\
& \leq \frac{C}{s+\delta}
\end{aligned}
$$

which implies a contradiction. 
Case 2: there is $\widetilde{\tau_{1}^{*}} \in\left(\tau_{1}, \tau_{2}\right]$ satisfying $S\left(\widetilde{\tau_{1}^{*}}\right) \leq C / s+\delta$. Since $S\left(\tau_{2}\right)>C / s \pm \delta$ and by the continuity of $S(\tau)$ in $\left(\tau_{1}, \tau_{2}\right]$, there is $\widetilde{\tau_{1}^{*}} \in\left[\widetilde{\tau_{1}^{*}}, \tau_{2}\right]$ satisfying

$$
S\left(\widetilde{\widetilde{\tau_{1}^{*}}}\right)=\frac{C}{s+\delta}, S(\tau)>\frac{C}{s+\delta}, \quad \tau \in\left(\widetilde{\tilde{\tau}_{1}^{*}}, \tau_{2}\right] .
$$

For $\tau \in\left(\widetilde{\tilde{\tau}_{1}^{*}}, \tau_{2}\right]$ and $\theta \in[-l, 0]$, we obtain

$$
(s+\delta) S(\tau)>S(\tau+\theta)
$$

By (51) and (ii), for $\tau \in\left(\widetilde{\tilde{\tau}_{1}^{*}}, \tau_{2}\right]$, we have

$$
D^{+} S(\tau) \leq M_{\beta}\left(\gamma-\mu_{j}\right) S(\tau) \leq 0 .
$$

Then,

$$
S\left(\tau_{2}\right) \leq S\left(\widetilde{\tau_{1}^{*}}\right)=\frac{C}{s},
$$

which is a contradiction. By induction, we can show that, for $p=1,2, \ldots$,

$$
S(\tau)<C, \quad \tau \in\left(\tau_{p-1}, \tau_{p}\right] .
$$

Finally, we can derive that, for $\tau \geq-l, S(\tau)<C$.

Proceeding as the proof of Theorem 1, we have $\forall \tau \geq 0$ and

$$
\mathbb{E}|\sigma(\tau)|^{q} \leq C_{1} \beta^{-\gamma}(\tau),
$$

where

$$
C_{1}=\frac{1}{1-2^{q-1} k^{q} \beta^{\gamma}(l)} \times 2^{q-1}\left(\frac{C}{\varepsilon}+k^{q} \beta^{\gamma}(l) \mathbb{E}\|\chi\|^{q}\right) .
$$

Therefore,

$$
\limsup _{\tau \rightarrow \infty} \frac{\ln \mathbb{E}\left(|\sigma(\tau)|^{q}\right)}{\ln \beta(\tau)} \leq-\gamma,
$$

as desired.

\section{Example}

Consider the following NISFDEwMS:

$$
\begin{cases}d(\sigma(\tau)-G(\sigma(\tau-1)))=f_{1}(\sigma(\tau-1), \tau, m(\tau)) \mathrm{d} \tau+f_{2}(\sigma(\tau-1), \tau, m(\tau)) \mathrm{d} w(\tau), & \tau \neq \tau_{p}, \\ \Delta \sigma\left(\tau_{p}\right)=-\epsilon^{p} \sigma\left(\tau_{p}\right)+\epsilon^{p} \sigma\left(\tau_{p}-1\right), \text { and } \tau_{p}=0.5 p, & p=1,2, \ldots \\ \sigma(\tau)=\chi, & \tau \in[-1,0]\end{cases}
$$

where

$$
\begin{aligned}
& f_{1}(\sigma(\tau-1), \tau, 1)=-\frac{\alpha_{1}}{1+\tau} \sigma(\tau)+\frac{\alpha_{2} \tau}{(1+\tau)^{2}} G(\sigma(\tau-1)), \\
& f_{1}(\sigma(\tau-1), \tau, 2)=\frac{1}{2} f_{1}(\sigma(\tau-1), \tau, 1), \\
& f_{2}(\sigma(\tau-1), \tau, 1)=\frac{\alpha_{3}}{1+\tau}(\sigma(\tau)-G(\sigma(\tau-1))), \\
& f_{2}(\sigma(\tau-1), \tau, 2)=\frac{1}{\sqrt{2}} f_{2}(\sigma(\tau-1), \tau, 1),
\end{aligned}
$$

where $\varepsilon>0$ is arbitrary and $l=1$. We assume that $\alpha_{1} \geq \alpha_{2}>0$ and $\alpha_{3} \in \mathbb{R}$ such that $\alpha_{1}>\alpha_{3}^{2} / 2, w(\tau)$ is standard one- dimensional Brownian Motion, and $G: P \mathscr{C}([-1,0]$, $\left.\mathbb{R}^{i}\right) \longrightarrow \mathbb{R}^{i}$ is Borel measurable such that $G(0)=0$, and there exists $k \in(0,1)$ such that

$$
\left|G\left(\psi_{1}\right)-G\left(\psi_{2}\right)\right| \leq k \sup _{-1 \leq \theta \leq 0}\left|\psi_{1}(\theta)-\psi_{2}(\theta)\right|,
$$

where $\forall \psi_{1}, \psi_{2} \in \operatorname{PC}\left([-1,0] \mathbb{R}^{i}\right)$ and

$$
\Gamma=\left(\begin{array}{cc}
-1 & 1 \\
1 & -1
\end{array}\right) .
$$

Let

$$
V(\sigma, \tau, 1)=V(\sigma, \tau, 2)=|\sigma|^{2},
$$

then

$$
\begin{aligned}
\mathscr{L} V(\sigma(\tau), \sigma(\tau-1), \tau, 1) & \leq 2(\sigma(\tau)-G(\sigma(\tau-1)))^{T}\left(\frac{-\alpha_{1}}{1+\tau} \sigma(\tau)+\frac{\alpha_{2} \tau}{\left(1+\tau^{2}\right)} G(\sigma(\tau-1))\right)+\frac{\alpha_{3}^{2}}{(1+\tau)^{2}}|\sigma(\tau)-G(\sigma(\tau-1))|^{2} \\
& \leq \frac{-2 \alpha_{1}}{1+\tau}(\sigma(\tau)-G(\sigma(\tau-1)))^{T}(\sigma(\tau)-G(\sigma(\tau-1)))+\frac{\alpha_{3}^{2}}{(1+\tau)^{2}}|\sigma(\tau)-G(\sigma(\tau-1))|^{2} \\
& \leq \frac{-1}{1+\tau}\left(2 \alpha_{1}-\alpha_{3}^{2}\right) V(\sigma(\tau)-G(\sigma(\tau-1)), \tau, 1) .
\end{aligned}
$$




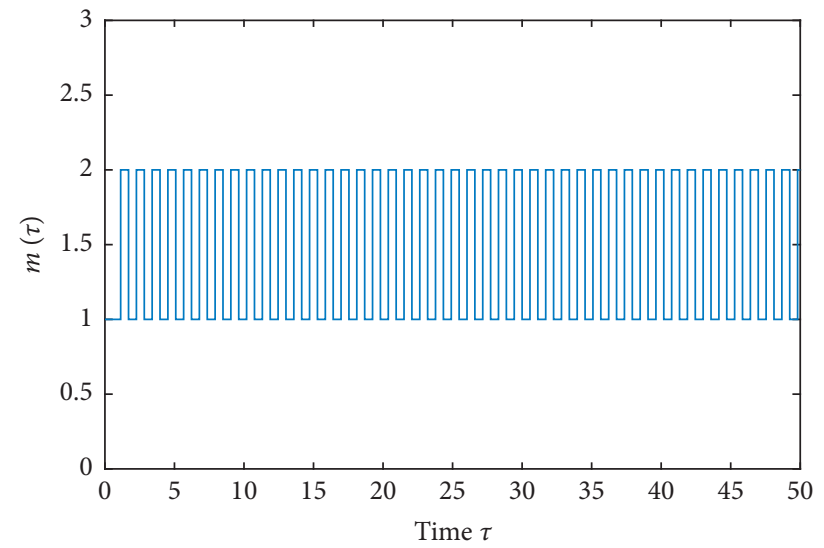

Figure 1: Response curve of Markovian switching in $m(\tau)$ in system (87).

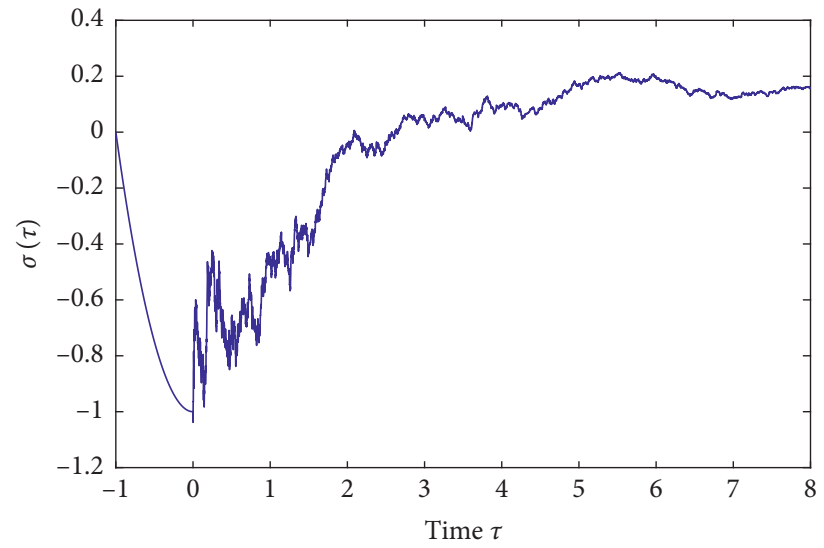

Figure 2: Response curve of system (87) without impulses with initial condition $\chi(\tau)=\tau^{2}-1$.

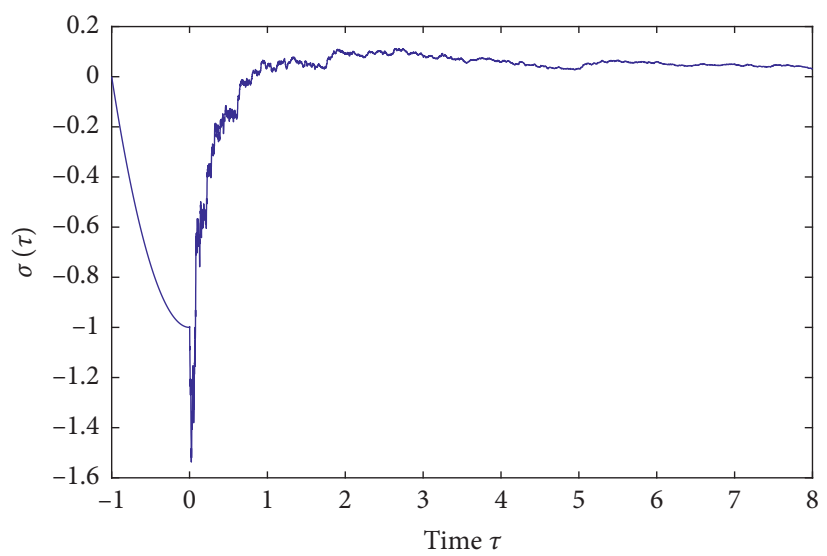

Figure 3: Response curve of system (87) with impulses with initial condition $\chi(\tau)=\tau^{2}-1$. 
Then,

$$
\mathbb{E}(\mathscr{L} V(\sigma(\tau), \sigma(\tau-1), \tau, 1)) \leq \frac{-1}{1+\tau}\left(2 \alpha_{1}-\alpha_{3}^{2}\right) \mathbb{E}(V(\sigma(\tau)-G(\sigma(\tau-1)), \tau, 1))
$$

Thus, $\mu_{1}=2 \alpha_{1}-\alpha_{3}^{2}$ and $\beta(\tau)=1+\tau$.

By the same computation, we have

$$
\mathbb{E}(\mathscr{L} V(\sigma(\tau), \sigma(\tau-1), \tau, 2)) \leq \frac{-1}{2(1+\tau)}\left(2 \alpha_{1}-\alpha_{3}^{2}\right) \mathbb{E}(V(\sigma(\tau)-G(\sigma(\tau-1)), \tau, 2))
$$

Thus, $\mu_{2}=1 / 2\left(2 \alpha_{1}-\alpha_{3}^{2}\right)$ and $\beta(\tau)=1+\tau$. For $j=1,2$,

$$
\begin{aligned}
& \mathbb{E}\left(V\left(\psi(0)-G(\psi)+I_{p}\left(\psi(0), \psi(\theta), \tau_{p}, j\right), \tau_{p}^{+}, j\right)\right) \\
& =\mathbb{E}\left|\sigma\left(\tau_{p}\right)-G\left(\sigma\left(\tau_{p}-1\right)\right)+\varepsilon^{p} \sigma\left(\tau_{p}-1\right)-\varepsilon^{p} \sigma\left(\tau_{p}\right)\right|^{2} \\
& =\mathbb{E}\left|\left(1-\varepsilon^{p}\right)\left(\sigma\left(\tau_{p}\right)-G\left(\sigma\left(\tau_{p}-1\right)\right)\right)+\varepsilon^{p}\left(\sigma\left(\tau_{p}-1\right)-G\left(\sigma\left(\tau_{p}-1\right)\right)\right)\right|^{2} \\
& \leq 2\left(1-\varepsilon^{p}\right)^{2} \mathbb{E}\left|\sigma\left(\tau_{p}\right)-G\left(\sigma\left(\tau_{p}-1\right)\right)\right|^{2}+2 \varepsilon^{2 p} \sup _{-1 \leq \theta \leq 0}\left(\mathbb{E}\left|\sigma\left(\tau_{p}+\theta\right)-G\left(\sigma\left(\tau_{p}+\theta\right)\right)\right|^{2}\right) \\
& =a_{j, p} \mathbb{E}\left(V\left(\sigma\left(\tau_{p}\right)-G\left(\sigma\left(\tau_{p}-1\right)\right), \tau_{p}, j\right)\right)+\bar{a}_{j, p} \sup _{-1 \leq \theta \leq 0}\left(\mathbb{E}\left(V\left(\sigma\left(\tau_{p}+\theta\right)-G\left(\sigma\left(\tau_{p}+\theta\right)\right), \tau_{p}+\theta, j\right)\right)\right),
\end{aligned}
$$

where $a_{1, p}=a_{2, p}=2\left(1-\varepsilon^{p}\right)^{2}$ and $\bar{a}_{1, p}=\bar{a}_{2, p}=2 \varepsilon^{2 p}$. Hence, we can choose $\lambda, \gamma$, and $\epsilon>0$ such that $\max _{1 \leq p<\infty}\left(1-\varepsilon^{p}\right)^{2}<$ $1 / 4, \max _{1 \leq p<\infty} \varepsilon^{2 p} 2^{\gamma}<1 / 4, \lambda \leq 0.5, \mu_{j}-\gamma>\ln (s) / \lambda$, and

$$
s=\frac{1}{\max _{j \in\{1,2\}, 1 \leq p<\infty}\left\{a_{j, p}+\bar{a}_{j, p} 2^{\gamma}\right\}}>1 .
$$

Then, system (87) is polynomially stable in mean square.

The response curve of the Markovian switching with $m(0)=1$ is given in Figure 1. Taking $G(x)=1 / 2 \sin (x)$ and choosing $\alpha_{1}=2, \alpha_{2}=1, \alpha_{3}=1$, and $\varepsilon=0.8$, we obtain the response curve of solution of system (87) in Figure 2. In Figure 3, it can be seen that continuous dynamics of system (87) with initial condition $\chi(\tau)=\tau^{2}-1$ can be successfully stabilized by delayed impulses.

From Figures 2 and 3, although neutral SFDEwMS without impulsive effect may be polynomially unstable in mean square, adding impulsive effect may become polynomially stable in mean square, which implies that impulsive effect may change the stable behavior of a system.

\section{Conclusion}

In this paper, $\beta$-stability in $q$-th moment for NISFDEwMS is obtained. For future work, we will try to obtain the $\beta$-stability of random impulsive stochastic pantograph differential equation.

\section{Data Availability}

The data used to support the findings of this study are available from the corresponding author upon request.

\section{Conflicts of Interest}

The authors declare that they have no conflicts of interest.

\section{Acknowledgments}

The authors extend their appreciation to the Deanship of Scientific Research at King Saud University for funding this work through research group (No. RG-1441-328).

\section{References}

[1] X. Mao, Stochastic Differential Equations and Applications, Horwood, Chichester, UK, 2 edition, 2008.

[2] X. Mao, Exponential Stability of Stochastic Differential Equations, Marcel Dekker, New York, NY, USA, 1994.

[3] X. Mao, "Robustness of exponential stability of stochastic differential delay equations," IEEE Transactions on Automatic Control, vol. 41, no. 3, pp. 442-447, 1996.

[4] H. Chen, P. Shi, and C.-C. Lim, "Stability of neutral stochastic switched time delay systems: an average dwell time approach," International Journal of Robust and Nonlinear Control, vol. 27, no. 3, pp. 512-532, 2017.

[5] H. Chen and L. Wang, "New result on exponential stability for neutral stochastic linear system with time-varying delay," 
Applied Mathematics and Computation, vol. 239, pp. 320-325, 2014.

[6] L. Huang and F. Deng, "Razumikhin-type theorems on stability of neutral stochastic functional differential equations," IEEE Transactions on Automatic Control, vol. 53, no. 7, pp. 1718-1723, 2008.

[7] T. Caraballo, M. A. Hammami, and L. Mchiri, "Practical exponential stability of impulsive stochastic functional differential equations," Systems \& Control Letters, vol. 109, pp. 43-48, 2017.

[8] H. Chen, P. Shi, C.-C. Lim, and P. Hu, "Exponential stability for neutral stochastic Markov systems with time-varying delay and its applications," IEEE Transactions on Cybernetics, vol. 46, no. 6, pp. 1350-1362, 2016.

[9] G. Hu and K. Wang, "Stability in distribution of neutral stochastic functional differential equations with Markovian switching," Journal of Mathematical Analysis and Applications, vol. 385, no. 2, pp. 757-769, 2012.

[10] X. Mao and C. Yuan, Stochastic Differential Equations with Markovian Switching, Imperial College Press, London, UK, 2006.

[11] L. Pan and J. Cao, "Exponential stability of stochastic functional differential equations with Markovian switching and delayed impulses via Razumikhin method," Advances in Difference Equations, vol. 61, pp. 1-18, 2012.

[12] W. Cao and Q. Zhu, "Razumikhin-type theorem for $p$ th exponential stability of impulsive stochastic functional differential equations based on vector Lyapunov function," Nonlinear Analysis: Hybrid Systems, vol. 39, p. 100983, 2021.

[13] G. Yu, W. Yang, L. Xu, H. Chen, and Y. Zhao, "Pth moment and almost sure exponential stability of impulsive neutral stochastic functional differential equations with Markovian switching," International Journal of Systems Science, vol. 49, no. 6, pp. 1164-1177, 2018.

[14] T. Caraballo, M. A. Hammami, and L. Mchiri, "Practical asymptotic stability of nonlinear stochastic evolution equations," Stochastic Analysis and Applications, vol. 32, no. 1, pp. 77-87, 2014.

[15] G. Pavlovic and S. Janković, "Razumikhin-type theorems on general decay stability of stochastic functional differential equations with infinite delay," Journal of Computational and Applied Mathematics, vol. 236, pp. 1679-1690, 2012.

[16] G. Pavlovic and S. Janković, "The Razumikhin approach on general decay stability for neutral stochastic functional differential equations," Journal of the Franklin Institute, vol. 350, pp. 2124-2145, 2013. 